\title{
Quantum state cloning using Deutschian closed timelike curves
}

\author{
Todd A. Brun \\ Ming Hsieh Department of Electrical Engineering, \\ University of Southern California, Los Angeles, California 90089, USA \\ Mark M. Wilde \\ Department of Physics and Astronomy, Center for Computation and Technology, \\ Louisiana State University, Baton Rouge, Louisiana 70803, USA \\ Andreas Winter \\ ICREA \& Física Teòrica: Informació i Fenomens Quàntics, \\ Universitat Autònoma de Barcelona, ES-08193 Bellaterra (Barcelona), Spain and \\ School of Mathematics, University of Bristol, Bristol BS8 1TW, United Kingdom
}

(Dated: October 29, 2018)

\begin{abstract}
We show that it is possible to clone quantum states to arbitrary accuracy in the presence of a Deutschian closed timelike curve (D-CTC), with a fidelity converging to one in the limit as the dimension of the CTC system becomes large - thus resolving an open conjecture from [Brun et al., Physical Review Letters 102, 210402 (2009)]. This result follows from a D-CTC-assisted scheme for producing perfect clones of a quantum state prepared in a known eigenbasis, and the fact that one can reconstruct an approximation of a quantum state from empirical estimates of the probabilities of an informationally-complete measurement. Our results imply more generally that every continuous, but otherwise arbitrarily non-linear map from states to states can be implemented to arbitrary accuracy with D-CTCs. Furthermore, our results show that Deutsch's model for CTCs is in fact a classical model, in the sense that two arbitrary, distinct density operators are perfectly distinguishable (in the limit of a large CTC system); hence, in this model quantum mechanics becomes a classical theory in which each density operator is a distinct point in a classical phase space.
\end{abstract}

PACS numbers: 03.65.Wj, 03.67.Dd, 03.67.Hk, 04.20.Gz

Keywords: no-cloning theorem, closed timelike curves, open timelike curves

The possible existence of closed timelike curves (CTCs) in certain exotic spacetime geometries [1-3] has sparked a significant amount of research regarding their ramifications for computation [4 6] and information processing [7. 8. One of the well known models for CTCs is due to Deutsch 9, who had the insight to abstract away much of the space-time geometric details and use the tools of quantum information to address physical questions about causality paradoxes. One consequence is that quantum computers with access to "Deutschian" CTCs (D-CTCs) would be able to answer any computational decision problem in PSPACE [6], a powerful complexity class containing the well-known class NP, for example. Also, quantum information processors with access to D-CTCs could distinguish non-orthogonal states perfectly [7, thus leading to the strongest violation of the uncertainty principle that one could imagine. From the perspective of Aaronson [10, 11, we might take these results to be complexity- and information-theoretic evidence against the existence of CTCs that behave according to Deutsch's model.

In order to avoid "grandfather-like" paradoxes, Deutsch's model imposes a boundary condition, in which the density operator of the CTC system before it has interacted with a chronology-respecting system should be equal to the density operator of the CTC system after it interacts. More formally, let $\rho_{S}$ denote the state of the chronology-respecting system and let $\sigma_{C}$ denote the state of the CTC system before a unitary interaction $U_{S C}$ (acting on systems $S$ and $C$ ) takes place. The first assumption of Deutsch's model is that the state of the chronology-respecting system $S$ and the chronologyviolating system $C$ is a tensor-product state, since presumably they have not interacted before the CTC system comes into existence. Furthermore, Deutsch's model imposes the following self-consistency condition:

$$
\sigma_{C}=\Phi_{\rho}\left(\sigma_{C}\right) \equiv \operatorname{Tr}_{S}\left\{U_{S C}\left(\rho_{S} \otimes \sigma_{C}\right) U_{S C}^{\dagger}\right\},
$$

so that potential grandfather paradoxes can be avoided. Computationally, one can take the view that nature is finding a fixed point of the map $\Phi_{\rho}[\underline{6}$, 9, which depends on the state $\rho_{S}$ of the chronology-respecting system. The chronology-respecting system's state evolves by

$$
\rho_{S} \rightarrow \rho_{\text {out }}=\operatorname{Tr}_{C}\left\{U_{S C}\left(\rho_{S} \otimes \sigma_{C}\right) U_{S C}^{\dagger}\right\},
$$

where the partial trace is over the CTC system. Since $\sigma_{C}$ depends on $\rho_{S}$, such an evolution is non-linear and as a result is a non-standard quantum evolution.

In developing the above consistency condition, Deutsch explicitly assumed that density operators are the fundamental object characterizing quantum systems, and, under this assumption, Deutsch's model does not lead to 
any of the classical time-travel paradoxes $[9$. If the density operator is viewed as a statistical ensemble or as a state of knowledge, then Deutsch's consistency condition becomes problematic and could conceal underlying paradoxes [9, 12.

Since quantum processors with access to D-CTCs can perfectly distinguish pure quantum states [7, one might conclude that such D-CTC-assisted processors could also approximately clone any pure quantum state, in violation of the celebrated no-cloning theorem [13, 14. In fact, Deutsch suggested that quantum cloning should be possible when one has access to D-CTCs behaving according to (1) 9], and Brun et al. conjectured that "a [D-CTC-assisted] party can construct a universal cloner with fidelity approaching one, at the cost of increasing the available dimensions in ancillary and CTC resources" 7]. Indeed, a simple idea for building an approximate cloner would be to discretize a given finite-dimensional Hilbert space, by casting an $\varepsilon$-net over all of the pure states in it, such that any state in the Hilbert space is $\varepsilon$-close in trace distance to a state in the $\varepsilon$-net. (Simple arguments for the size of such $\varepsilon$-nets are well known [15].) One would then construct a unitary for perfectly distinguishing states in the $\varepsilon$-net, according to the procedure given in [7, and produce clones according to the classical outcome of the distinguishing device. States in the $\varepsilon$-net would be cloned perfectly, while the hope is that states that are not in the $\varepsilon$-net would be identified with the closest state in the $\varepsilon$-net.

An approach similar to this was pursued in [16, and the numerical evidence given there suggests that such an approach should work in general. However, it is well known (and perhaps obvious) that there are continuity issues with D-CTCs 6, 9, 17, so that one cannot easily appeal to continuity in order to develop this argument in greater detail.

In this paper, we give an approach to quantum state cloning with D-CTCs that is conceptually different from the aforementioned one, and it is also significantly simpler and thus more appealing. We show how to clone any quantum state, such that the fidelity of each clone approaches one as the dimension of the assisting D-CTC system becomes large. An important implication of our result is that Deutsch's model turns quantum theory into a classical theory, in the sense that each density operator becomes a distinct, distinguishable point in a classical phase space.

One can quickly grasp the main idea behind our construction by taking a glance at the circuit in Figure 1 . The first step is to perform an informationally-complete measurement on the incoming state $\rho_{S}$. Such a measurement is well known in quantum information theory [1820] - the probabilities of the outcomes are in one-to-one correspondence with a classical density operator description of the quantum state. (That is, if one knew these probabilities, or could estimate them from performing this kind of measurement on many copies of the given state, then one could construct a classical description of the state.) Let $\omega$ denote the state resulting from the measurement:

$$
\rho \rightarrow \sum_{x=0}^{d-1} \operatorname{Tr}\left\{M_{x} \rho\right\}|x\rangle\langle x| \equiv \omega,
$$

where each $M_{x}$ is an element of the informationallycomplete measurement (so that $M_{x} \geq 0$ for all $x$ and $\left.\sum_{x} M_{x}=I\right), d$ is the number of possible measurement outcomes, and $\{|x\rangle\}$ is the standard computational basis.

Next, we feed the state $\omega$ into a circuit that cyclically permutes it with $N$ CTC systems that each have the same dimension as $\omega$. Such an operation on its own (after tracing over all systems except for the $N$ CTC systems) has as its unique fixed point the state $\omega^{\otimes N}$, so that, in some sense, the cyclic shift produces $N$ "temporary" clones.

Finally, we copy the value of $x$ from each of the $N$ CTC systems to one of a set of ancillary systems in order to "read out" $N$ copies of the state $\omega$. In Figure 1 we have depicted this operation as a sequence of controlled-not (CNOT) gates, but in fact it will generally be a higherdimensional analogue of a CNOT, like a modular addition circuit:

$$
|x\rangle|y\rangle \rightarrow U(|x\rangle|y\rangle)=|x\rangle|(x+y) \bmod d\rangle .
$$

The fixed point of the overall circuit, after tracing over all systems except for the $N$ CTC systems, is still $\omega^{\otimes N}$, because these modular addition gates do not cause any disturbance to the CTC systems. As a result, the reduced state on the $N$ ancillas is equal to $\omega^{\otimes N}$, and we can then estimate the eigenvalues of $\omega$ simply by counting frequencies - the estimates become better and better as $N$ becomes larger due to the law of large numbers. Since these eigenvalues result from an informationallycomplete measurement, we can construct a classical description of the state $\rho$ and produce as many approximate copies of it as we wish.

We now develop this argument in more detail. We first show how to produce perfect clones of a quantum state that is diagonal in a known eigenbasis. Suppose that the initial state of the system and the CTC is as follows:

$$
\rho_{S} \otimes \sigma_{C}
$$

where $S$ is a $d$-dimensional system and $C$ consists of $N$ $d$-dimensional systems. Furthermore, let $\rho_{S}$ have the following spectral decomposition:

$$
\rho_{S}=\sum_{x} p_{X}(x)|x\rangle\left\langle\left. x\right|_{S},\right.
$$

where $p_{X}(x)$ is a probability distribution and $\left\{|x\rangle_{S}\right\}$ is some orthonormal basis. The first operation is to perform a cyclic shift by one to the right of all $N+1$ systems, i.e., 


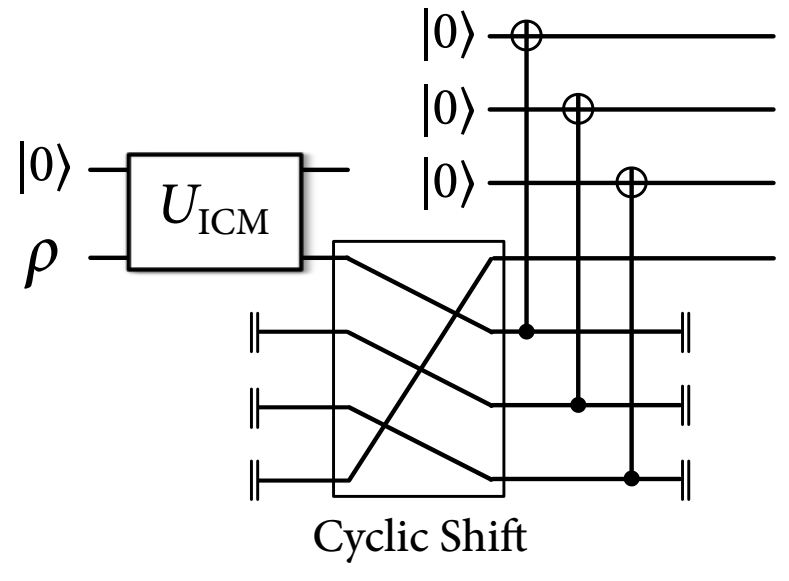

FIG. 1: Example circuit for cloning using $N=3$ CTC systems. An unknown state $\rho$ is fed into a unitary $U_{\mathrm{ICM}}$, whose effect is to implement an informationally-complete measurement with operators $\left\{M_{x}\right\}$, such that $M_{x} \geq 0$ and $\sum_{x} M_{x}=I$. The resulting state $\omega=\sum_{x} \operatorname{Tr}\left\{M_{x} \rho\right\}|x\rangle\langle x|$ is combined with $N$ CTC systems and cyclically permuted with them. (For each CTC system, the past mouth of its wormhole on the left, indicated by vertical double lines, is identified with its future mouth on the right.) Finally, modular addition circuits (depicted here as CNOT gates) "read out" $N$ copies of the state $\omega$, from which we can estimate the original state $\rho$ to arbitrarily good accuracy as the number $N$ of CTC systems becomes large (of course, one would require $N$ to be much larger than three). The main text provides details of why this approach works for D-CTC systems.

the following unitary operation:

$$
\begin{aligned}
& \left|x_{1}\right\rangle_{S} \otimes\left|x_{2}\right\rangle_{C_{1}} \otimes\left|x_{3}\right\rangle_{C_{2}} \otimes \cdots \otimes\left|x_{N+1}\right\rangle_{C_{N}} \\
& \quad \rightarrow\left|x_{N+1}\right\rangle_{S} \otimes\left|x_{1}\right\rangle_{C_{1}} \otimes\left|x_{2}\right\rangle_{C_{2}} \otimes \cdots \otimes\left|x_{N}\right\rangle_{C_{N}},
\end{aligned}
$$

where we have broken up the system $C$ into $N$ parts as $C_{1} \cdots C_{N}$. One can then easily prove that, if this is the only interaction, the self-consistent and unique solution is for the CTC systems to be in the state $\rho^{\otimes N}$. Indeed, we can do so by demonstrating that $\rho^{\otimes N}$ is the unique fixed point of the above map. For simplicity, let us initialize the state of the CTC system so that it is maximally mixed, and so that the overall state is

$$
\rho_{S} \otimes \pi_{C_{1}} \otimes \pi_{C_{2}} \otimes \cdots \otimes \pi_{C_{N}},
$$

where $\pi$ is the maximally mixed qudit state. After a cyclic shift, the state becomes

$$
\pi_{S} \otimes \rho_{C_{1}} \otimes \pi_{C_{2}} \otimes \cdots \otimes \pi_{C_{N}} .
$$

Tracing over the system $S$ gives

$$
\rho_{C_{1}} \otimes \pi_{C_{2}} \otimes \cdots \otimes \pi_{C_{N}} .
$$

This becomes the initial state of the CTC for the next application of the map, so that the overall state is now

$$
\rho_{S} \otimes \rho_{C_{1}} \otimes \pi_{C_{2}} \otimes \cdots \otimes \pi_{C_{N}} .
$$

Applying the cyclic shift again gives $\pi_{S} \otimes \rho_{C_{1}} \otimes \rho_{C_{2}} \otimes \cdots \otimes$ $\pi_{C_{N}}$, so that the reduced state is $\rho_{C_{1}} \otimes \rho_{C_{2}} \otimes \cdots \otimes \pi_{C_{N}}$. It is then clear that applying the above procedure $N$ times in total gives the following state for the CTC

$$
\rho_{C_{1}} \otimes \rho_{C_{2}} \otimes \cdots \otimes \rho_{C_{N}}
$$

and further applications will not change anything, so that this is the fixed point of the CTC. (In fact, by taking an arbitrary initial state for the CTC, we can easily see by a similar procedure that the state in (11) will be the unique fixed point after applying the map $N$ times.)

Now, this procedure already produces $N$ temporary clones of the initial state, and one might claim that this circuit on its own is a cloner. However, the $N$ clones in the CTC systems are not available after these systems enter the future mouth of the wormhole, so that this cloner is not particularly useful. We would like to have a circuit for which the clones are available after the CTC systems are no longer in existence.

Since we have assumed for now that we know the eigenbasis of the incoming state, there is a simple modification of the above circuit that will allow for cloning it. Consider again performing the circuit given above. As we showed, the fixed point solution for the CTC is $\rho^{\otimes N}$. What we can do after the cyclic shift is to copy the value of $x$ from the $N$ CTC systems to $N d$-dimensional ancilla states initialized to the state $|0\rangle$, by using a modular addition circuit. These circuits perform the unitary in (3) in the eigenbasis of the incoming system; they therefore cause no disturbance to the CTC systems, and the fixed point solution for the state of the CTC is still $\rho^{\otimes N}$. Furthermore, the marginal state on the ancillas and the original system is $\rho^{\otimes N+1}$, so that we have successfully produced $N$ clones of the state of the incoming system, in the case where its eigenbasis is known. (If the eigenbasis is not known, then one can easily check that our circuit will decohere the incoming state $\rho$ in the basis in which the modular addition circuits are specified and produce $N$ perfect copies of the decohered state.)

The above circuit allows for perfect cloning of quantum states in a known eigenbasis. A particular preprocessing of an arbitrary incoming state will allow us to produce approximate clones whose fidelity with the incoming state becomes arbitrarily high in the limit where the number $N$ of CTC systems becomes large. Let $\rho$ denote the density operator of the input state. We can perform a measurement map of the form in (2) on the incoming state. Such a map is a CPTP map, so that we can perform it by first appending an ancilla of sufficient size, acting with a unitary on the joint system, and tracing out the ancilla. We should be sure to choose the measurement map to be informationally complete, such that the outcome probabilities are in one-to-one correspondence with the parameters of the density operator.

The procedure for approximate cloning is as follows: 
1. On the incoming state, perform the measurement map specified by (2).

2. Append the $N$ CTC systems to this state and send the $N+1$ systems through the cyclic shift circuit, followed by $N$ CNOT gates from the CTC systems to $N$ ancilla systems.

3. The resulting state after the CTC expires is $\omega^{\otimes N+1}$ (with $\omega$ defined in (2)).

4. Perform measurements in the basis $\{|x\rangle\}$ to estimate the distribution $\operatorname{Tr}\left\{M_{x} \rho\right\}$ to arbitrarily good accuracy (with $N$ large).

5. Based on the estimate, produce as many approximate clones of $\rho$ as desired.

In Step 4, we can argue that the estimate becomes arbitrarily good as $N$ becomes large, due to the law of large numbers. In particular, Hoeffding's bound states that the probability for the empirical frequencies to deviate from their true values by more than any constant $\delta>0$ is bounded from above as $2 \exp \left\{-2 N \delta^{2}\right\}$ [21], so that this probability rapidly converges to zero as the number $N$ of CTC systems increases. The number of CTC systems scales well with the desired accuracy for cloning (and the number of gates is linear in the number of CTC systems) - to have an estimation error no larger than some constant $\varepsilon>0$ requires a number of gates no larger than $O(\log (1 / \varepsilon))$.

A slight modification of the above protocol would be to avoid tracing over the ancilla after performing the unitary corresponding to the measurement map. The state resulting from the unitary is $\sum_{x, y}\left(M_{x} \rho M_{y}^{\dagger}\right)_{E} \otimes|x\rangle\left\langle\left. y\right|_{B}\right.$ if the input state is $\rho$, where we have labeled the environment as $E$ and the output as $B$. We could then append $N$ CTC systems, labeled as $E_{1} B_{1} \cdots E_{N} B_{N}$ that are each the same dimension as the composite system $E B$. After that, we would perform a cyclic shift of the $E_{i} B_{i}$ systems, followed by a CNOT gate from each $B_{i}$ system to an external ancilla. It is straightforward to show that the fixed-point solution of the CTC systems $E_{1} B_{1} \cdots E_{N} B_{N}$ is then $\left(\sum_{x} M_{x} \rho M_{x}^{\dagger} \otimes|x\rangle\langle x|\right)^{\otimes N}$. That is, the effect of the CNOT gates is to decohere the $B_{i}$ systems. The CNOT gates will then read out many copies of the state $\sum_{x} \operatorname{Tr}\left\{M_{x} \rho\right\}|x\rangle\langle x|$ to the external ancillas, from which we can estimate the input state $\rho$ as before. An advantage of this approach is that this modified circuit avoids potential interpretational issues with the initial measurement map. That is, one might claim that the measurement map in (2) actually "collapses" the state $\rho$ to one of the states $|x\rangle$ with probability $\operatorname{Tr}\left\{M_{x} \rho\right\}$ and the resulting circuit merely copies the given state $|x\rangle$ many times, providing no advantage for cloning over an ordinary quantum circuit. However, by having all evolutions be unitary, it is clear that the modified circuit avoids this interpretational problem.
By a well-known argument [14, the ability to clone implies the ability to signal superluminally, so that this is the case for our cloner here (assuming the usual description of quantum measurements). Our results imply more generally that every continuous, but otherwise arbitrarily non-linear map $f$ from states to states can be implemented to arbitrary accuracy with Deutschian CTCs. This follows because we can estimate the incoming state $\rho$ to arbitrary accuracy and then prepare $f(\rho)$ at will.

Discussion - An "open timelike curve" is one in which a quantum system enters the future mouth of a wormhole and emerges from the past mouth of the wormhole without ever interacting with itself along the way [8]. Our circuit in Figure 1 indicates that we are very close to implementing quantum state cloning using only an open timelike curve. If the modular addition circuits were not present, then this approach would indeed be just an open timelike curve. We say that we are "very close" because in our setup, the modular addition circuits do not disturb the state of the CTC systems, so one might be tempted to expand the definition of an open timelike curve to allow for such non-disturbing interactions.

One might question the method above by taking an adversarial approach to quantum state cloning as was done with quantum state discrimination in 22. In such an adversarial model as described in [22], an adversary would prepare a labeled mixture of states of the form $\sum_{x} p(x)|x\rangle\langle x| \otimes \rho_{x}$, feed in the second system to the cloner, and demand that the output state of the composite system be $\sum_{x} p(x)|x\rangle\langle x| \otimes \tilde{\rho}_{x} \otimes \tilde{\rho}_{x}$, where $\tilde{\rho}_{x}$ is a good approximation to $\rho$. Our approach will not satisfy this demand but instead outputs an approximate copy of the average state $\sum_{x} p(x) \rho_{x}$ because Deutsch's criterion in (1) stipulates that the fixed point is computed with respect to the reduced state of the system entering the CTC device. However, such behavior is to be expected, since quantum mechanics in Deutsch's model is no longer linear, so that the action of a map on a mixture of states is not equal to the mixture of states resulting from the map acting on each state. Some authors have argued that it is not sensible to represent ensembles as labeled mixtures when we are dealing with a non-linear theory 23. Labeled mixtures are in one-to-one correspondence with ensembles in standard quantum mechanics, but this correspondence breaks down in a non-linear theory. One might also argue that all of this points to the Deutsch model itself being incomplete 24. Regardless, what we have shown in this paper is that if a quantum state $\rho$ is presented to a device that behaves according to the prescription of Deutsch's model, then it is possible to produce an arbitrary number of very good approximate clones of $\rho$.

Our results imply that, in a particular sense, Deutsch's model is actually a classical model for CTCs rather than a quantum model. That is, quantum theory supplemented with Deutsch's prescription for CTCs seems to require 
an interpretation as a classical probability theory on the space of density operators. This in turn leaves open the question of whether other descriptions of CTCs might retain more of the distinctive features of quantum theory. This feature of Deutsch's model originates from the way that it combines quantum features (density operators and unitary evolutions) with non-quantum ones (non-linear evolution) in an ad hoc way. In the framework of generalized probabilistic theories 25 27, there is a basic result stating that, if states and evolutions are defined operationally, then the evolution must be linear in the state [28. The fact that Deutsch's model allows for evolutions that are non-linear in the density operator implies that the set of all density operators corresponds to linearly independent states at the operational level. Our result strengthens this observation for the case of Deutsch's model, showing that the states corresponding to density operators are not only linearly independent, but even perfectly distinguishable.

Open Questions - It might be considered somewhat unsatisfactory that we obtain only an arbitrarily good approximation of cloning. The limit achieving perfect cloning requires taking the size of both the CTC system and the ancillary system to infinity. This seems to be necessary if we would like to read out the parameters of the density operator as we have done here, but this is less clear if all we desire is to have two clones of the incoming state. So an open question to consider going forward from here is if there exists an exact $1 \rightarrow 2$ CTC-assisted cloner that requires only a finite external system but with a potentially infinite internal CTC system.

We are grateful to Patrick Hayden and John Preskill for insightful discussions and especially to the anonymous referees for insightful comments and additions. MMW is grateful for the hospitality of the Ming Hsieh Department of Electrical Engineering at the University of Southern California and Física Teòrica: Informació i Fenomens Quàntics at the Universitat Autònoma de Barcelona and for support for research visits during April and May 2013. AW acknowledges financial support from the Spanish MINECO (project FIS2008-01236), the European Commission (STREP "QCS"), the ERC (Advanced Grant "IRQUAT") and a Philip Leverhulme Prize.

[1] Kurt Gödel. An example of a new type of cosmological solutions of Einstein's field equations of gravitation. Reviews of Modern Physics, 21(3):447-450, July 1949.

[2] J. Richard Gott. Closed timelike curves produced by pairs of moving cosmic strings: Exact solutions. Physical Review Letters, 66(9):1126-1129, March 1991.

[3] William B. Bonnor. The rigidly rotating relativistic dust cylinder. Journal of Physics A: Mathematical and General, 13(6):2121, 1980.

[4] Todd A. Brun. Computers with closed timelike curves can solve hard problems. Foundations of Physics Letters, 16:245-253, 2003. arXiv:gr-qc/0209061.

[5] Dave Bacon. Quantum computational complexity in the presence of closed timelike curves. Physical Review A, 70(3):032309, September 2004. arXiv:quant-ph/0309189.

[6] Scott Aaronson and John Watrous. Closed timelike curves make quantum and classical computing equivalent. Proceedings of the Royal Society A, 465(2102):631647, 2009. arXiv:0808.2669.

[7] Todd A. Brun, Jim Harrington, and Mark M. Wilde. Localized closed timelike curves can perfectly distinguish quantum states. Physical Review Letters, 102(21):210402, May 2009. arXiv:0811.1209.

[8] Jacques L. Pienaar, Timothy C. Ralph, and Casey R. Myers. Open timelike curves violate Heisenberg's uncertainty principle. Physical Review Letters, 110:060501, February 2013. arXiv:1206.5485.

[9] David Deutsch. Quantum mechanics near closed timelike lines. Physical Review D, 44(10):3197-3217, November 1991.

[10] Scott Aaronson. NP-complete problems and physical reality. ACM SIGACT News, 36(1):30-52, March 2005. arXiv:quant-ph/0502072.

[11] Scott Aaronson. Quantum computing, postselection, and probabilistic polynomial-time. Proceedings of the Royal Society A, 461(2063):3473-3482, 2005. arXiv:quant$\mathrm{ph} / 0412187$.

[12] Joel J. Wallman and Stephen D. Bartlett. Revisiting consistency conditions for quantum states of systems on closed timelike curves: An epistemic perspective. Foundations of Physics, 42(5):656-673, May 2012. arXiv:1005.2438.

[13] William K. Wootters and Wojciech H. Zurek. A single quantum cannot be cloned. Nature, 299:802803, 1982.

[14] Dennis Dieks. Communication by EPR devices. Physics Letters A, 92(6):271272, 1982.

[15] Patrick Hayden, Debbie Leung, Peter W. Shor, and Andreas Winter. Randomizing quantum states: Constructions and applications. Communications in Mathematical Physics, 250(2):371-391, 2004. arXiv:quant-ph/0307104.

[16] Doyeol Ahn, Casey R. Myers, Timothy C. Ralph, and Robert B. Mann. Quantum state cloning in the presence of a closed timelike curve. 2012. arXiv:1207.6062.

[17] Richard DeJonghe, Kimberly Frey, and Tom Imbo. Discontinuous quantum evolutions in the presence of closed timelike curves. Physical Review D, 81:087501, April 2010. arXiv:0908.2655.

[18] Eduard Prugovecki. Information-theoretical aspects of quantum measurement. International Journal of Theoretical Physics, 16:321-331, May 1977.

[19] Paul Busch. Informationally complete sets of physical quantities. International Journal of Theoretical Physics, 30(9):1217-1227, September 1991.

[20] Joseph M. Renes, Robin Blume-Kohout, A. J. Scott, and Carlton M. Caves. Symmetric informationally complete quantum measurements. Journal of Mathematical Physics, 45:21712180, 2004. arXiv:quant-ph/0310075.

[21] Wassily Hoeffding. Probability inequalities for sums of bounded random variables. Journal of the American Statistical Association, 58(301):1330, March 1963.

[22] Charles H. Bennett, Debbie Leung, Graeme Smith, and John A. Smolin. Can closed timelike curves or nonlinear quantum mechanics improve quantum state discrimination or help solve hard problems? Physical Review 
Letters, 103(17):170502, October 2009. arXiv:0908.3023.

[23] Eric G. Cavalcanti and Nicolas C. Menicucci. Verifiable nonlinear quantum evolution implies failure of density matrices to represent proper mixtures. 2010. arXiv:1004.1219.

[24] Eric G. Cavalcanti, Nicolas C. Menicucci, and Jacques L. Pienaar. The preparation problem in nonlinear extensions of quantum theory. 2012. arXiv:1206.2725.

[25] Lucien Hardy. Quantum theory from five reasonable axioms. 2001. arXiv:quant-ph/0101012.

[26] Jonathan Barrett. Information processing in generalized probabilistic theories. Physical Review A, 75:032304,
March 2007. arXiv:quant-ph/0508211.

[27] Giulio Chiribella, Giacomo Mauro D'Ariano, and Paolo Perinotti. Probabilistic theories with purification. Physical Review A, 81:062348, June 2010. arXiv:0908.1583.

[28] "Linear" is meant here in the operational sense: a state is a linear combination of a set of states if the outcome probabilities for that state are linear combinations of the outcome probabilities of those states, for every possible measurement; relative to this operational notion of linearity, a physical transformation must send a linear combination of input states to a linear combination of output states. 